\title{
In Hospital Clinical Outcome of Percutaneous Coronary Intervention (PCI) Result of 150 Cases
}

Mr. Jamal Uddin, Mahboob Ali, AHK Chowdhury, AAS Majumber, N. Hossain, Khondker Q. Islam, M. Raisuddin, F. Rahman, Muhammad Abdus Salam, Kaiser Nasrulla Khan, SK. Marufuzzaman, Kazi Abul Hasan, Khaled Mohsin, Atahar Ali, Imran, Asm Rabban, Nazrul Islam, H. P. Nepal

This is a ongoing study done in National Institute of Cardiovascular Diseases (NICVD), Dhaka, Bangladesh during the period May 2002 to June 2003. Total number of study population was 150 with male $135 \&$ female 15 . Indication of percutaneous coronary intervention (PCl) was Unstable angina with no prior myocardial infarction 60 cases. Myocardial infarction (MI) inferior with postmyocardial infarction angina 40 cases \& $\mathrm{Ml}$ anterior with post $\mathrm{Ml}$ angina 50 cases. The risk factors were Hypertension (HTN) in 72 cases $(48 \%)$, smoking in 90 cases (60\%), Diabetes Mellitus (DM) in 50 cases (33.33\%), Positive family history for ischemic heart disease (IHD) in 40 cases $(26.66 \%)$ and Dyslipidemia 35 cases $(23.32 \%)$, Total number of target coronary arteries were 190 of which 184 lesions treated. Distribution of 184 lesions were left anterior descending coronary artery (LAD) 91 (Proximal LAD 40, mid LAD 40, distal LAD 11), Right coronary artery (RCA) 60 (Proximal 15, mid RCA 40, distal RCA 05), Left circumflex coronary artery (LCX) 26 (Proximal LCX 05, mid LCX 15, distal LOX 04, LCX PD 02), Diagonal 02, Obtuse marginal (OM) 04, Ramus intermedius 02.

Angiographic diagnosis of the patients were single vessel disease $113(75.32 \%)$, Double vessel disease 22 (14.66\%), Triple vessel disease 15 (10\%); No. of lesions treated was 184 , of which 160 PTCA with stenting \& 24 cases plain PTCA done. Total occlusion was treated in 25 cases. The native vessel has a mean reference diameter was $0.03 \pm 0.53$ $\mathrm{mm}$. Residual stenosis after deploying stents was $<10 \%$. Our success rate wasangiographic success $96 \%$, procedural success $94 \%$ \& clinical success $90 \%$. Failad PCl in 6 cases all of them were chronic total occlusion. Two patients died, one of them died 
one hour after the procedure due to sudden asystole. The patient was female with triple vessel disease. Another one died due to acute occlusion of both LAD \& LCX.

In hospital course of the patients were uneventful. All the patients were discharged by three to fourth day of the procedure with improvement of their clinical condition. 